\title{
FREDHOLM-VOLTERRA INTEGRAL EQUATION IN THE CONTACT PROBLEM
}

\author{
A.A. El-Bary \\ Basic and Applied Science Department, \\ Arab Academy for Science and Technology, \\ P.O. Box 1029, Alexandria, Egypt
}

\begin{abstract}
In this paper, under certain condition the Fredholm-Volterra integral equation of the first kind is solved. The existence and uniqueness of the solution is considered. The Fredholm integral equation of the second kind is established from the work, and its solution is also obtained.
\end{abstract}

Keywords-Fredholm-Volterra integral equation, Logarithmic kernel, Legendre polynomial.

\section{INTRODUCTION}

Singular integral equations arises in many problems of mathematical physics, in the theory of elasticity, viscoelasticity, hydrodynamics, biological problems, population, genetics, and others. On the other hand, many authors are interested in studying the different methods for solving the singular integral equation, (see [1,19,25]).

Over the past thirty years substantial progress has been made in developing innovative approximate analytical and purely numerical solution to a large class of singular integral equation. The solution of these problems can be obtained analytically, using the theory developed by Muskhelishvili [15,16]. The books edited by Green [2], Hochstad [7], Popov [5] and Tricomi [4] contained many different methods to solve the integral equation analytically. The books edited by Golberg $[13,14]$ contain extensive literature surveys on approximate, analytical and purely numerical techniques. The interested reader should consult the fine expositions by Atkinson [10], Delves and Mohammed [12] and Linz [27] for numerical methods solution. Kovalenko [3] obtained a Fredholm integral equation of the first kind with singular kernel, when the mixed problems of continuous media with boundary conditions specified on a circle is studied.

In [16] Abdou solved the Fredholm integral equation of the second kind, which is investigated from the semi-symmetric Hertz problem for two different elastic materials in three dimensions. The solution of the three dimensional contact problem of two different elastic material obtained, and the structure resolvent is, also, established in the domain of contact $\Omega=(x, y): \sqrt{x^{2}+y^{2}} \leq a, z=0$ in the work of Abdou [15]. Kauthen in his work: [9] used a collection method to solve the Fredholm-Volterra integral equation numerically. In [11] a projection method based on the Fourier theory is used to obtain an approximate solution for linear Volterra-Fredholm integral equation of the second kind. Burner, in his work [6], used collection method to obtain, numerically, the solution of nonlinear Volterra-Fredholm integral equation.

In this work, the Fredholm-Volterra integral equation is considered under certain conditions, and the solution in series form of a Legendre polynomial is obtained. The 
existence and uniqueness of the solution is taken in mind. The convergence of the series is discussed.

\section{FORMULATION OF THE PROBLEM}

Consider an elastic material of strip $(\mathrm{G}, v)$ of thickness $h$. Occupying the region $0 \leq y \leq h,|x| \prec \infty$, lies without friction on rigid elastic layer surface $h(x)$ describing it. Consider a rigid rectangular stamp of width $2 \mathrm{a}$ is impressed into the boundary of the strip $\mathrm{y}=\mathrm{h}$, through a function of time $\mathrm{F}(\mathrm{t}), 0 \leq t \leq T \prec \infty$, by a constant force $p \prec \infty$, whose eccentricity of application e. Here $v$ and $G$ are the Poisson's coefficient and the displacement module of each material, respectively.

Assume the frictional forces in the contact area between the stamp and the strips are so small that it can be neglected.

It is known [15] that such a problem leads us to the following boundary value problem:

$$
\begin{aligned}
& \lambda \int_{-1}^{1} K\left(\frac{\xi-x}{\lambda_{1}}\right) \phi(\xi, \mathrm{t}) d \xi+\lambda \int_{0}^{t} \mathrm{~F}(\tau) \phi(x, \tau) d \tau=\pi[\delta(\mathrm{t})+\alpha(t) x-h(x)]=\pi \mathrm{f}(\mathrm{x}, \mathrm{t}) \\
& |x| \leq 1, t \in[0, T], \lambda \text { is a constant } \lambda_{1} \in[(0, \infty)] \\
& K(y)=\frac{1}{2} \int_{-\infty}^{\infty} \frac{\tanh S(u)}{S(u)} e^{i u y} d u, \quad\left(y=\frac{\xi-x}{\lambda}\right)
\end{aligned}
$$

under the conditions:

$$
p(t)=\int_{-1}^{1} \phi(\xi, t) d \xi, \quad \mathrm{M}(t)=\int_{-1}^{1} \xi \phi(\xi, t) d \xi
$$

Here $\delta(t)$ is the rigid displacement under the action of a variable force $P(t)$ and $\alpha(t)$ are the twisting of $\mathrm{M}(\mathrm{t}), 0 \leq t \prec T \prec \infty$.

\section{THE SINGULAR KERNEL OF THE PROBLEM}

Formula (2.2) can be written in the form (see [5])

$$
\mathrm{K}(\mathrm{y})=\frac{1}{2} \int_{-\infty}^{\infty} \frac{\tanh \mathrm{S}(\mathrm{u})}{\mathrm{S}(\mathrm{u})} \mathrm{e}^{\mathrm{iuy}} \mathrm{du}=-\ln \left|\tanh \frac{\pi \mathrm{y}}{4}\right|, \mathrm{y}=\frac{\xi-\mathrm{x}}{\lambda_{1}}
$$


when $\lambda_{1} \longrightarrow \infty, \mathrm{y}$ is sufficiently small, that it satisfies tanh $\mathrm{y} \approx \mathrm{y}$, we have

$\ln \left|\tanh \frac{\pi(\xi-x)}{4 \lambda_{1}}\right|=\ln |\xi-x|-d ; \quad\left(d=\ln \frac{4 \lambda_{1}}{\pi}\right)$

Substituting from (3.1) and (3.2) in equation (2.1), then we have:

$\lambda \int_{-1}^{1}[-\ln |\xi-\mathrm{x}|+\mathrm{d}] \phi(\xi, \mathrm{t}) \mathrm{d} \xi+\lambda \int_{0}^{\mathrm{t}} \mathrm{F}(\tau) \phi(\mathrm{x}, \tau) \mathrm{d} \tau=\pi \mathrm{f}(\mathrm{x}, \mathrm{t})$

\section{METHOD OF SOLUTION}

If we divide the interval $[0, T], o \leq t \leq T \prec \infty$, in the following form $0 \leq \mathrm{t}_{\mathrm{o}} \prec \mathrm{t}_{1} \prec \mathrm{t}_{2} \ldots . \prec \mathrm{t}_{\mathrm{n}}=\mathrm{T}$, i.e $\mathrm{t}=\mathrm{t}_{\mathrm{k}}, \mathrm{k}=1,2, \ldots \ldots . \mathrm{N}$. ,

the second integral of $(3.3)$ with the quadratic formula $u_{j}(j=1,2, \ldots . k)$ gives

$\int_{o}^{t_{k}} F(\tau) \phi(x, \tau) d \tau=\sum_{j=0}^{k} u_{j} F\left(t_{j}\right) \phi\left(x, t_{j}\right)+0\left(\hbar^{p}\right) \quad(p \geq k)$

where

$\hbar_{k}=\max _{0 \leq t \leq k} h_{j}, h_{j}=t{ }_{j+1}^{-t} j$

The characteristic points of (4.1) with the quadratic coefficients $u_{\mathrm{j}}$ are explained in [12]. Hence (3.3) becomes

$\lambda \int_{-1}^{1}[-\ln |\xi-x|+d] \phi\left(\xi, t_{k}\right) d \xi+\lambda \sum_{j=0}^{k} u_{j} F\left(t_{j}\right) \phi\left(x, t_{j}\right)=\pi f\left(x, t_{k}\right)$

$$
\mu_{\mathrm{k}} \phi_{\mathrm{k}}(\mathrm{x})+\lambda \int_{-1}^{1}\left[-\ln |\xi-\mathrm{x}| \phi_{\mathrm{k}}+\mathrm{d}\right] \phi_{\mathrm{k}}(\xi) \mathrm{d} \xi+\lambda \sum_{\mathrm{j}=0}^{\mathrm{k}-1} \mathrm{u}_{\mathrm{j}} \mathrm{F}_{\mathrm{j}} \phi_{\mathrm{j}}(\mathrm{x})=\pi \mathrm{f}_{\mathrm{k}}(\mathrm{x})
$$

The formula (4.2) can be adapted in the form where

$$
\mu_{k}=\lambda u_{k} F_{k}
$$

The general solution of (4.3) can be obtained by using the recurrence relation, for example at $\mathrm{k}=0$, we have the integral equation

$$
\lambda \int_{-1}^{1}[-\ln |\xi-x|+d] \phi_{0}(\xi) d \xi=\pi f_{0}(x)
$$

Many different methods are used to solve equation (4.4) Abdou and Hassan [18] used potential theory method to obtain the function $\phi_{0}(x)$ which is the solution of (4.4) when the given function takes a Chebychev polynomial form. Also in [17] Abdou and EzzEldin, solved the integral equation (4.4) when the free function takes a Chebychev 
polynomial form by using Krein's method. In [16] Abdou obtained and derived many spectral relations in the form of eigenvalues and eigenfunction of the formula (4.4) the leader work for the different methods to solve (4.4) can be found in [26] in the form:

$$
\phi_{0}(x)=\frac{1}{\pi \lambda_{1} \sqrt{1-x^{2}}}\left[P+\frac{1}{\pi} \int_{-1}^{1} \sqrt{\frac{1-\tau^{2}}{\tau-x}} f_{0}^{\prime}(\tau) d \tau\right]
$$

where

$f_{0}^{\prime}(y)=\frac{d f_{0}(y)}{d y}, \quad P=\int_{-1}^{1} \phi_{0}(x) d x$

Let $\mathrm{k}=1$, in (4.3), we have

$\mu_{1} \phi_{1}(\mathrm{x})+\lambda \int_{-1}^{1}[-\ln |\xi-\mathrm{x}|+\mathrm{d}] \phi(\xi) \mathrm{d} \xi+\lambda \mathrm{u}_{0} \mathrm{~F}_{0} \phi_{0}(\mathrm{x})=\pi \mathrm{f}_{1}(\mathrm{x})$

to solve the integral equation (4.5), we differentiate it with respect to $\mathrm{x}$, to get

$\mu_{1} \frac{\mathrm{d} \phi_{1}}{\mathrm{dx}}-\lambda \int_{-1}^{1} \frac{\phi_{1}(\xi) \mathrm{d} \xi}{\xi-\mathrm{x}}=\pi \mathrm{g}_{1}(\mathrm{x})$

where

$g_{1}(x)=f_{1}(x)-\frac{\lambda}{\pi} u_{0} F_{0} \phi_{0}(x)$

Assume the solution of (4.7) in the Legendre polynomials form

$\phi_{1}(x)=\sum_{n=0}^{\infty} C_{n}^{(l)} P_{n}(x)$

Where $C_{n}^{(1)}$ are constants and $P_{n}(x)$ are the Legendre polynomials. If $\phi_{1}(x) \in L_{2}(-1,1)$, the polynomial series of (4.8) is also convergent.

The Legendre polynomials satisfies the orthogonal relation form (see [8])

$$
\int_{-1}^{1} P_{n}(x) P_{m}(x) d x=\left\{\begin{array}{cc}
0 & m \neq n \\
\frac{2}{2 n+1} & m=n
\end{array}\right.
$$

Differentiating (4.8) with respect to $\mathrm{x}$, we have

$$
\phi_{1}^{\prime}(x)=\sum_{n=0}^{\infty} C_{n}^{(1)} P_{n}^{(1)}(x) \cdot\left(1-\mathrm{x}^{2}\right)^{-1 / 2}
$$

Also, the free term $g_{1}(x)$ can be represented in the form 


$$
g_{1}(x)=\sum_{n=0}^{\infty} g_{n}^{(1)} P_{n}^{(1)}(x) \cdot\left(1-\mathrm{x}^{2}\right)^{-1 / 2}
$$

Where $g_{n}^{1}$ are constant terms, which can be determined by using the orthogonal relation. If $g(x) \in L_{2}(-1,1)$, the polynomial series (4.11) is also convergent to $g(x)$ in $L_{2}(-1,1)$. While $\mathrm{P}_{\mathrm{n}}^{(\mathrm{m})}(\mathrm{x})$ is the associated Legendre polynomial of the first kind, which satisfies the orthogonal relation.

$$
\int_{-1}^{1} \mathrm{P}_{\mathrm{n}}^{\mathrm{k}}(\mathrm{x}) \mathrm{P}_{\mathrm{m}}^{\mathrm{k}}(\mathrm{x}) \mathrm{dx}=\left\{\begin{array}{cc}
0 & ; \mathrm{n} \neq \mathrm{m} \\
\frac{2}{2 \mathrm{n}+1} \cdot \frac{(\mathrm{n}+\mathrm{k}) !}{(\mathrm{n}-\mathrm{k}) !} & ; \mathrm{n}=\mathrm{m}
\end{array}\right.
$$

(see [8] pp. 808)

Hence, the integral term of (4.7) can be written in the form

$$
\int_{-1}^{1} \frac{\phi_{1}(\xi)}{x-\xi} d \xi=2 \sum_{n=0}^{\infty} \mathrm{C}_{n}^{1} Q_{n}(x)
$$

(See [8] pp. 835)

Where

$$
Q_{n}(x)=\frac{1}{2} \int_{-1}^{1} \frac{P_{n}(y)}{x-y} d y
$$

In view of equation (4.11), the values of $\mathrm{g}_{n}(1)$ of (4.11) takes the form

$$
g_{n}^{(1)}=\frac{-(2 n+1)}{2 n(n+1)} \int_{-1}^{1} g_{1}(x) \sqrt{1-x^{2}} P_{n}^{1}(x) d x
$$

Hence, substituting from equations. (4.10), (4.11) and (4.13) in the integral equation (4.7), we have

$$
2 \lambda \sqrt{1-x^{2}} \sum_{n=0}^{\infty} C_{n}^{(1)} Q_{n}(x)=\sum_{n=0}^{\infty}\left(\pi g_{n}^{(1)}+\mu_{1} C_{n}^{(1)}\right) P_{n}^{1}(x)
$$

(4.16) Multiplying both sides of equation (4.16) by $P_{m}^{1}(x) d x$ then integrating form -1 to 1 and with the aid of the following integral relation (see [8] pp. 807) 


$$
\int_{-1}^{1} \sqrt{1-x^{2}} Q_{n}(x) P_{m}^{1}(x) d x=\left\{\begin{array}{cl}
0 & (n=m \neq 1) \\
\frac{-2(m+1)\left[1+(-1)^{n+m}\right]}{(m-n-1)(m-n+1)(m+n)(m+n+2)} & (n \neq m \neq 1)
\end{array}\right.
$$

The formula (4.16) takes the form

$$
\mu_{1} \mathrm{C}_{\mathrm{m}}^{(1)}+2 \sum_{\mathrm{n}=0}^{\infty} \frac{(2 \mathrm{~m}+1)\left[1+(-1)^{\mathrm{n}+m}\right] \mathrm{C}_{\mathrm{n}}^{(1)}}{(\mathrm{m}-\mathrm{n}-1)(\mathrm{m}-\mathrm{n}+1)(\mathrm{m}+\mathrm{n})(\mathrm{m}+\mathrm{n}+2)}=\pi \mathrm{g}_{\mathrm{m}}^{(1)}
$$

The infinite system (4.18) can be represented in the two infinite system of even and odd case,

where

$$
\mu_{1} X_{2 m}-\sum_{n=1}^{\infty} b_{2 m, 2 n} x_{2 n}=\pi G_{2 m}-b_{2 m, 0} P
$$

(for even function).

$$
\mu_{1} X_{2 m-1}-\sum_{n=1}^{\infty} b_{2 m+1,2 n-1} X_{2 n-1}=\pi G_{2 m-1}
$$

(for odd function)

where

$$
\begin{aligned}
& b_{m, n}=\frac{2(2 n+1)\left[1+(-1)^{n+m}\right]}{(m-n-1)(m-n+1)(m+n)(m+n+2)} \\
& b_{m, m-1}=\mathrm{b}_{\mathrm{m}, \mathrm{m}+1}=o
\end{aligned}
$$

For the convergence of the infinite series (4.19) we must have the inequality (see [8]).

$$
\sum_{n=1}^{\infty}\left|b_{m, n}\right| \prec S \quad(\mathrm{~m} \geq 1)
$$

Where

$$
S=\frac{3}{4}-4 \sum_{n=3}^{\infty} \frac{2 n+1}{n(n-2)(n+1)(n+3)}
$$

$$
\text { when } n \rightarrow \infty \text { in (4.20), we have } S_{\infty}=3 / 4
$$

Similarly, at $\mathrm{k}=2$, we have an integral equation: 
$\mu_{2} \phi_{2}+\lambda \int_{-1}^{1}[-\operatorname{Ln}|\xi-\mathrm{x}|+\mathrm{d}] \phi_{1}(\xi) \mathrm{d} \xi=\pi \mathrm{f}_{1}(\mathrm{x})-\mathrm{u}_{0} \mathrm{~F}_{0} \phi_{0}(\mathrm{x})-\mathrm{u}_{1} \mathrm{~F}_{1} \phi_{1}(\mathrm{x})$

Differentiating equation (4.22), we have

Let $\phi_{2}(\mathrm{x})=\sum_{\mathrm{n}=0}^{\infty} \mathrm{C}_{\mathrm{n}}^{(2)} \mathrm{P}_{\mathrm{n}}(\mathrm{x})$

By following the same previous method, we get

$$
2 \sqrt{1-x^{2}} \sum_{n=0}^{\infty} C_{n}^{(2)} Q_{n}(x)=\sum_{n=0}^{\infty}\left(\pi g_{n}^{(2)}+\mu_{1} C_{n}^{(2)}-u_{0} F_{0} C_{n}^{(1)}\right) P_{n}^{(1)}(x)
$$

Multiplying both sides of equation (4.24) by $P_{n}^{(1)} \mathrm{dx}$ and integrating through the interval $[-1,1]$, then using the relation (4.16), we obtain

$\mu_{2} C_{m}^{(2)}-u_{1} F_{1} C_{m}^{(1)}+2 \sum_{n=0}^{\infty} \frac{(2 m+1)\left[1+(-1)^{n+m}\right] C_{n}^{(2)}}{(m-n-1)(m-n+1)(m+n)(m+n+2)}=\pi g_{m}^{(2)}$

where $\mathrm{C}_{\mathrm{m}}^{(0)}$ is given by (4.17)

In general, we get for $(m \geq 1)$, the following:

$\mu_{k} \mathrm{C}_{\mathrm{m}}^{(\mathrm{k})}-\sum_{\mathrm{j}=1}^{\mathrm{k}-1} \mathrm{U}_{\mathrm{j}} \mathrm{F}_{\mathrm{m}}^{(\mathrm{j})}+2 \sum_{\mathrm{n}=0}^{\infty} \frac{\left.(2 \mathrm{~m}+1)\left[1+(-1)^{\mathrm{n}+\mathrm{m}}\right]\right]_{\mathrm{n}}^{\mathrm{C})}}{(\mathrm{m}-\mathrm{n}-1)(\mathrm{m}-\mathrm{n}+1)(\mathrm{m}+\mathrm{n})(\mathrm{m}+\mathrm{n}+2)}=\pi \mathrm{g}_{\mathrm{m}}^{(\mathrm{k})}, \quad(\mathrm{k} \neq 0)$

Acknowledgment-I would like to thank Prof. M.A. Abdou for his helpful discussions through this work.

\section{REFERENCES}

[1] A.C. Koya and F. Erdogan, On the Solution of Integral Equation with a Generalized Cauchy Kernel, Quart. Appl. Math. XLV, No. 3, 455-469, 1997

[2] C.D. Green, Integral Equation Methods, New York, 1969.

[3] E.V. Kovalenko, Some Approximate Methods of Solving Integral Equations of Mixed Problems. Appl. Math. Mech. 53, No, 1,1989.

[4] F.G. Tricomi, Integral Equations, Dover, New York, 1985.

[5] G.Ya. Popov, Contact Problems for a Linearly Deformable Functions, KievOdessa,1982.

[6] H. Brunner, On the Numerical Solution of Nonlinear Volterra-Fredholm Integral Equation by Collocation Methods, SIAM J. Number. Anal. 27, No. 4, 987-1000,1990.

[7] H. Hochstadt, Integral Equations, Wiley, New York, 1973.

[8] I.C. Gradchtein and I.M. Reguk, Integrals Tables, Summation, Series and Derivatives, Fizamatgiz, Moscow 1971. 
[9] J.P. Kauthen, Continuos Time Collection Method for Volterra-Fredholm Integral Equations, Numerische Math. 56, 409-424, 1989.

[10] K.E. Atkinson, A survey of Numerical Methods for the Solution of Fredholm Integral Equation of the Second Kind, SIAM, Philadelphia ,1976.

[11] L. Hocia, On Approximate Solution for Integral Equation of the Mixed Type, ZAMM 76,1996.

[12] L.M. Delves and J.L. Mohamed, Computational Methods for Integral Equations, Cambridge , 1985.

[13] M.A. Golberg., Solution Methods for Integral Equations, New York ,1979.

[14] M.A. Golberg, Numerical Solution of Integral Equations, New York ,1990.

[15] M.A. Abdou, Fredholm Integral Equations with Potential kernel and its Structure Resolvent, Appl. Math. Comp. 117,169-180,1998.

[16] M.A. Abdou, Fredholm Integral Equation of the Second Kind with Potential Kernel, Comp. Appl. Math. 72 ,161-167, 1996.

[17] M.A. Abdou and N.Y. Ezz-Eldin, Kreins Method with certain kernel for solving the Integral Equation of the First Kind, Periedica. Math. Hung. 28. No 2,143-149,1994. [18] M.A. Abdou and S.A. Hassan, Boundary Value of a Contact Problem, P.U.M.A. 5 No. 3, 311-316, 1994.

[19] N.I. Muskhelishvili, Some Basic Problems of Mathematical Theory of Elasticity, Noordroff, Holand ,1953.

[20] N.I. Muskhelishvili, Singular Integral Equations, Noordroff ,1953.

[21] V.M. Aleksandrov, E.V. Kovalenko and S.M. Makhitrain, On a Method of Obtaining Spectral Relationships for Integral Operators of Mixed Problems of Mechanics of Continuous Media, Prikl. Math. Mech. 46, 825-832, 1983.

[22] V.M. Aleksandrov, E.V. Kovalenko; On a Class of Integral Equations in the Displacement Problem in Continuous Mechanics, Dokl. Akad. Nauk. USSR 252,324$328,1980$.

[23] V.M. Aleksandrov, E.V. Kovalenko, Mathematical Method in Contact Problems, Inzh, Zh, Mekh. Tverd. Tela. No. $2,77-89,1984$.

[24] V.M. Aleksandrov, E.V. Kovalenko, Problems with mixed boundary conditions in continuous mechanics, Nauka Moscow 1986.

[25] S.M. Makhitariam, A special relationship in spheroidal wave functions and its application to contact problem, Appl. Math. Mech. 48, 611-618, 1984.

[26] S.M. Makhitariam and M.A. Abdou, On some of different methods for solving the Fredholm Integral Equation of the first kind with logarithmic Kernel Dokl. Akad. Nauk. Armenia 90,1-10, 1990.

[27] P. Linz, Analytical and Numerical methods for Volterra equations, SIAM, Philadelphia ,1985. 